Original article

\title{
Evaluation of CZT SPECT imaging for cardiac sympathetic innervation in healthy individuals and patients with atrial fibrillation
}

\author{
Stanislav M. Minin, Nikita A. Nikitin, Vitaly V. Shabanov, Denis V. Losik, Igor L. Mikheenko, Dmitry N. Ponomarev, \\ Evgeny A. Pokushalov, Alexander B. Romanov
}

Meshalkin National Medical Research Center, Novosibirsk, Russia

Received 14 April 2018, Revised 18 May 2018, Accepted 24 May 2018

(C) 2018, Minin S.M., Nikitin N.A., Shabanov V.V., Losik D.V., Mikheenko I.L., Ponomarev D.N., Pokushalov E.A., Romanov A.B.

(C) 2018, Russian Open Medical Journal

\begin{abstract}
Background - Cadmium zinc telluride (CZT) solid-state detectors have been recently introduced in the field of nuclear medicine in cardiology. The aim of the current study was to investigate the scintigraphic patterns of total uptake and the regional distribution of iodine-123 metaiodobenzylguanidine $\left({ }^{123} \mathrm{I}-\mathrm{mIBG}\right)$ in healthy volunteers and patients with atrial fibrillation (AF) using a gamma camera equipped CZT detectors.

Material and Methods - The total number of included subjects was 30 (15 patients with a paroxysmal form of AF and 15 healthy volunteers). Using a novel single-photon emission computed tomography (SPECT) cardiac dedicated gamma camera equipped CZTdetectors the scintigraphy was performed 15 minutes and 4 hours after intravenous administration of norepinephrine structural analogue ${ }^{123}$ I-mIBG. Thus, data from early and delayed phases were obtained. The heart-to-mediastinum ratio of ${ }^{123}$ I-mIBG uptake at 15 minutes (HMRe) and 4 hours (HMRd), as well as the washout rate (WR) and summed ${ }^{123}$ I-mIBG score (SMSe, SMSd) were measured.

Results - Patients and volunteers showed comparable results of the median ratio of ${ }^{123}$ I-mIBG uptake in the heart and mediastinum (HMR) in the early and delayed phases ( $p>0.05)$. In $14(93 \%)$ patients with $A F,{ }^{123}$ I-mIBG uptake defects in LV myocardium were detected as compared with $8(53 \%)$ healthy individuals $(p<0.05)$. The healthy volunteers showed no significant ${ }^{123} \mathrm{I}-\mathrm{mIBG}$ uptake defect in delayed scans compared with a median result in patients with $A F(p<0.001)$.

Conclusion - SPECT gamma camera with CZT-detectors is suitable to obtain qualitative tomographic slices of the myocardium and to assess regional changes in sympathetic innervation of the heart, which can be the early signs of an imbalance in the autonomic nervous system. Therefore, this camera may be useful for managing patients with various forms of AF.
\end{abstract}

Keywords: iodine-123, cardiac mIBG imaging, CZT SPECT, sympathetic nervous system, atrial fibrillation.

Cite as Minin SM, Nikitin NA, Shabanov VV, Losik DV, Mikheenko IL, Ponomarev DN, Pokushalov EA, Romanov AB. Evaluation of CZT SPECT imaging for cardiac sympathetic innervation in healthy individuals and patients with atrial fibrillation. Russian Open Medical Journal 2018 ; 7: e0308.

Correspondence to Nikita A. Nikitin. Address: Rechkunovskaya str., 15, Novosibirsk, 630055, Russia. Tel.: +7 923 1096609. Email: n_nikitin@outlook.com.

\section{Introduction}

Atrial fibrillation (AF) is the most common tachyarrhythmia in clinical practice, creating a high risk of stroke, thromboembolic and cardiac complications [1-2]. It is known that the autonomic nervous system (ANS) of the heart and its sympathetic division, in particular, is one of the key elements in the onset and maintenance of AF [3-4]. This is confirmed by a number of experimental studies, which showed a decrease in the inducibility of AF after denervation of the heart [5-7]. In addition, the effect on the ANS in the interventional treatment of AF increases the effectiveness of ablation procedure [8-9].

To date, in radiological practice, cardiac imaging with a neuronal tracer, in particular, with metaiodobenzylguanidinelabelled with iodine-123 ( $\left.{ }^{123} \mathrm{I}-\mathrm{mIBG}\right)$, is actively used for non-invasive evaluation of the state of sympathetic cardiac activity [10]. This tracer is a structural analogue of norepinephrine and reflects the process of its uptake, storage, and release in sympathetic nerve-endings [12]. The use of cardiac single-photon emission computed tomography (SPECT) with ${ }^{123}$ I-mIBG allows one to evaluate regional presynaptic reuptake of catecholamines, the functional state of sympathetic endings, and indirectly to estimate the density of adrenoreceptor of the heart [13].

As a general rule, indicators of planar scintigraphy and regional ${ }^{123}$ I-mIBG uptake, in a less degree, [14] are used to assess changes in sympathetic activity. This is due to the need to introduce large doses of tracer and to increase the duration of the SPECT data acquisition on Anger cameras (with $\mathrm{Nal}$-based crystals). However, in recent years, this problem has been solved through the development and introduction into clinical practice of gamma cameras equipped with semiconductor cadmium-zinc-tellurium (CZT) detectors [15]. Due to the high sensitivity of the detectors and the higher spatial resolution, it became possible to obtain qualitative tomographic sections of the heart and to estimate the regional ${ }^{123} \mathrm{I}$-mIBG uptake. 
Table 1. Baseline characteristics

\begin{tabular}{lcc}
\hline Characteristics & Paroxysmal AF $(n=15)$ & Volunteers $(n=15)$ \\
\hline Age, years & $59(49,64)$ & $31(26,41)$ \\
Females, $\mathrm{n}$ & $8(53 \%)$ & $7(47 \%)$ \\
Body mass index, $\mathrm{kg} / \mathrm{m}^{2}$ & - & $24(21,26)$ \\
Smoking, $\mathrm{n}$ & - & $4(27 \%)$ \\
AF duration, months & $42(28,72)$ & - \\
Hypertension, $\mathrm{n}$ & $11(73 \%)$ & - \\
Diabetes, $\mathrm{n}$ & $2(13 \%)$ & - \\
Ejection fraction, \% & $66(59,68)$ & - \\
Left atrium, cm & $5.4(5.2,5.7)$ & - \\
CHA2DS2-VASC score: & & $15(100 \%)$ \\
-0 & $3(20 \%)$ & - \\
-1 & $4(27 \%)$ & - \\
-2 & $5(33 \%)$ & - \\
-3 & $1(7 \%)$ & - \\
-4 & $2(13 \%)$ & - \\
\hline
\end{tabular}

Continuous data are presented as medians with interquartile range Me (IQR). Categorical variables are presented as numbers and percent no. (\%). AF, atrial fibrillation.

At the same time, only a few studies have been published in the world literature devoted to the scintigraphic study of violations of sympathetic innervation of the myocardium in patients with heart rhythm disorders and the use of CZT technologies [16, 17].

The aim of present study was to assess the scintigraphic patterns of total uptake and the regional distribution of ${ }^{123}$ I-mIBG in healthy volunteers and patients with paroxysmal AF with a gamma camera equipped with CZT-detectors.

\section{Material and Methods \\ Patients}

15 patients (8 women) with a paroxysmal AF aged $59(48,64)$ years and 15 healthy volunteers ( 7 women) aged $31(26,40)$ years were included in the study. The baseline characteristics of patients and volunteers are presented in Table 1.

The median duration of AF history in the patient's group was $42(28,72)$ months. In $8(53.3 \%)$ patients, the $\mathrm{CHA}_{2} \mathrm{DS}_{2}$-VASc score was $\geq 2$. Subsequently, patients with $\mathrm{AF}$ had a catheter ablation performed according to the guidelines [2].

The study was carried out in accordance with the standards of GCP (Good Clinical Practice) and the principles of the Declaration of Helsinki. The study protocol was approved by the expert council and the local ethics committee of the Meshalkin National Medical Research Center (Novosibirsk, Russia). Prior to enrollment, written informed consent was obtained from all patients. The study was registered in the international database (NCT02914860) and is part of a project supported by a grant from the Russian Science Foundation №17-75-20118.

\section{${ }^{123}$ I-mIBG imaging}

The study protocol included ${ }^{123} \mathrm{I}$-mIBG intravenous administration at a dose of $370 \pm 10 \% \mathrm{MBq}$ and the conduct of acquisition in 15 minutes (early study) and 4 hours after the injection (delayed study). All patients underwent thyroid uptake blockade using Lugol's solution for 3 days before the study and 3 days after the study with ${ }^{123}$ I-mIBG (5 drops of Lugol's solution 3 times a day).

Cardiac scintigraphy was performed on a gamma camera with solid-state detectors based on cadmium-zinc-tellurium crystals (DSPECT, Spectrum Dynamics Medical Inc.). Data acquisition using 9 moving low-energy wide-angle collimators, a spatial resolution of $8.6 \mathrm{~mm}$ (voxel size $4.92 \times 4.92 \times 4.92 \mathrm{~mm}$ ). The centre of the energy window was set to a photopeak of ${ }^{123} \mathrm{I}-159 \pm 10 \mathrm{keV}$. The width of the energy window was symmetrical and amounted to $10 \%$. The collection time was 600 seconds. Correction for attenuation and dispersion was not carried out. The total effective dose was 6.0-7.3 $\mathrm{mSv}$. Images were reconstructed in a workstation using the OSEM iterative reconstruction algorithm. The analysis of the obtained images was carried out using dedicated software packages: PlanarH2M (Spectrum Dynamics Medical Inc.) for calculating heart to mediastinum ratio, quantitative perfusion SPECT (QPS) applications (Cedar Sinai Auto Quant, QBS, QPS v7.2) for semiquantitative determination of the regional ${ }^{123}$ I-mIBG uptake in the left ventricular (LV) myocardium [18] (Figure 1).

The sympathetic activity of the heart was assessed by calculating the ratio of ${ }^{123} \mathrm{I}-\mathrm{mIBG}$ uptake in the heart and mediastinum in the early and delayed phases (heart mediastinum ratio early and delayed, $\mathrm{H} / \mathrm{Me}$ and $\mathrm{H} / \mathrm{Md}$ ), as well as washout rates (WR) for 4 hours. A semiquantitative assessment of the regional ${ }^{123}$ I-mIBG uptake was performed using sections along the short and long (horizontal and vertical) axis of the heart, and a 17segmented polar LV map evaluated in the early and delayed acquisitions (summed mIBG score early - SMSe, and delayed SMSd) [15-16]. The results of the computer reconstruction of LV slices along a short axis were represented in the form of a polar chart of "bull's eye" conventionally divided into 17 segments, in each segment the degree of ${ }^{123} \mathrm{I}$-mIBG uptake was estimated as a percentage of the maximum recorded. The degree of ${ }^{123}$ I-mIBG uptake was estimated using a 5-point scale [19].

\section{Statistical analysis}

Continuous data are presented as medians with interquartile range - Me (IQR). Independent samples were compared with Wilcoxon rank sum test. Wilcoxon signed rank test was used for paired samples. Categorical variables are presented as numbers and percent - no. (\%), comparisons were done with Fisher's exact test. To assess associations between two continuous variables, linear regression was used; results are presented as regression estimate (i.e. a measure of association between the outcome and predictor), associated $95 \%$ confidence interval $(95 \% \mathrm{Cl})$ and $\mathrm{p}$ value for a trend. Missing values were not imputed. All the presented $p$-values were based on a two-sided test and a $p$-value $<0.05$ was considered statistically significant.

\section{Results}

The median ratio of ${ }^{123}$ I-mIBG uptake in the heart and mediastinum in the early (heart mediastinum ratio early, HMRe) and delayed (heart mediastinum ratio delayed, HRMd) phase was comparable in patients and volunteers: HRMe $-1.73(1.61,1.83)$ and $1.61(1.56,1.70)$, respectively, $p=0.265$; HMRd $-1.81(1.63$, $1.95)$ and $1.67(1.64,1.71), p=0.164$. The median washout rate of the ${ }^{123}$ I-mIBG (WR) was slightly lower in patients compared to volunteers: $17.8(16.7,20.5)$ and $22.5(18.3,28.1)$, respectively $p=0.159)$. 

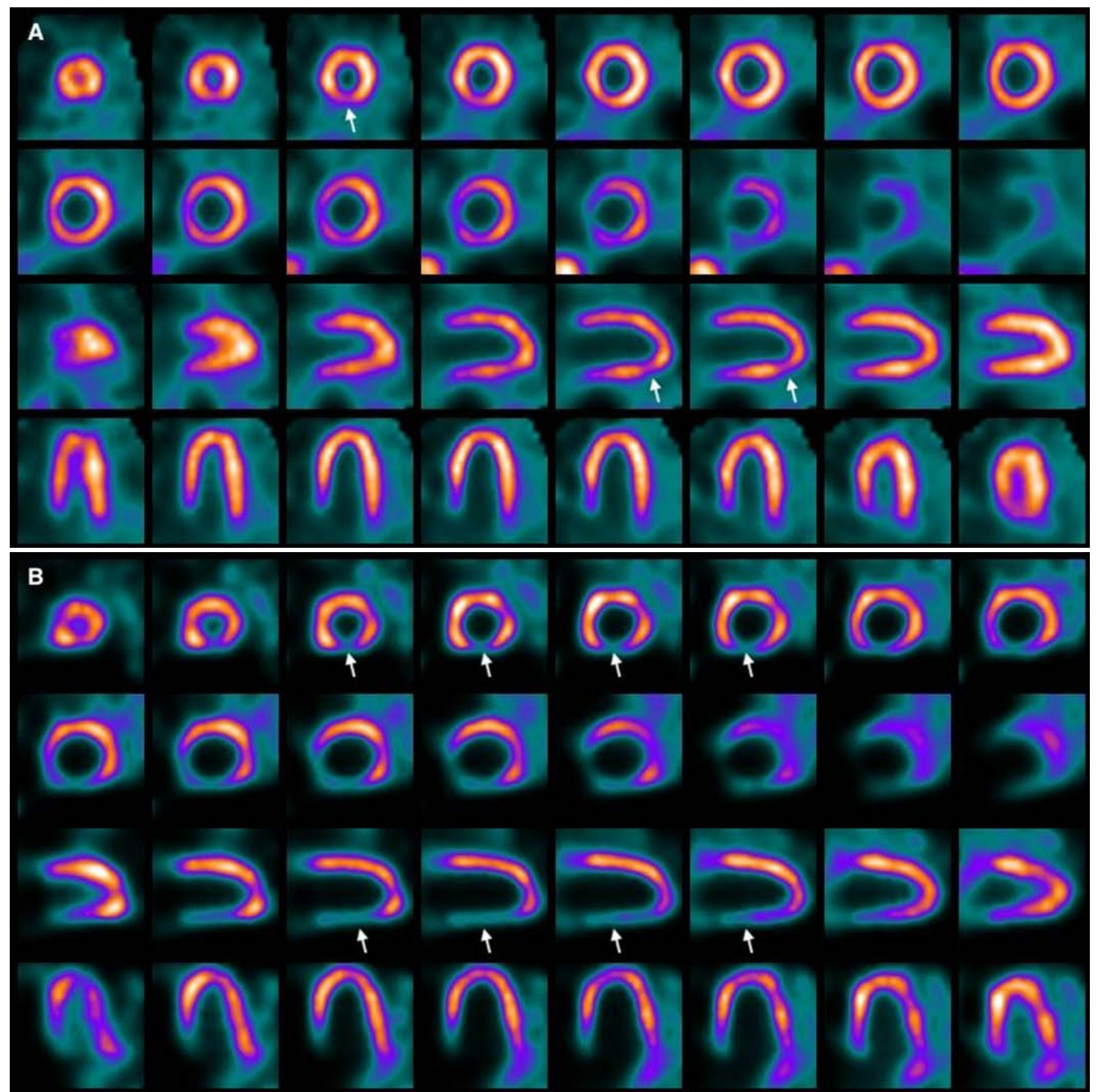

Figure 1. Short- and long-axis slices of the left ventricle 4 hours after the ${ }^{123} \mathrm{I}-\mathrm{mIBG}$ administration in a healthy individual (A) and AF patient (B). ${ }^{123} \mathrm{I}-\mathrm{mIBG}$ uptake defects in a healthy individual and an AF patient are indicated with white arrows.

In 14 (93\%) patients with $\mathrm{AF},{ }^{123} \mathrm{I}$-mIBG uptake defects in LV myocardium were detected as compared with $8(53 \%)$ healthy individuals, $p=0.035$. The most frequent defects of ${ }^{123} \mathrm{I}$-mIBG uptake were found in the inferior LV wall (in patients with $A F$ in $78.6 \%$ of cases, in healthy individuals in $66 \%$ of cases) and at the apex of the LV (in patients with AF in $64.2 \%$ of cases, in healthy individuals in $77.7 \%$ of cases).

In the group of patients with paroxysmal AF, the median ${ }^{123} \mathrm{I}-$ mIBG uptake defect in early scans (summed mIBG score early, SMSe) was significantly lower than that in delayed scans (summed
mIBG score delayed, SMSd): $0(0,1)$ vs $3(1,4), p=0.008$ (Figures $2 a$ and $2 \mathrm{~b}$ ). In the group of healthy individuals, the median SMSe was $0(0,0)$ and remained unchanged on repeat scan (Figures $3 a$ and $3 b)$. The median SMSe was similar in the two groups: $0(0,1)$ and 0 $(0,0)$ in patients and volunteers, respectively, $p=0.237$. By contrast, the median SMSd was significantly higher in patients compared with volunteers: $3(1,4)$ and $0(0,0), p<0.001$.

In the AF patients, there was a non-significant negative association between $\mathrm{WR}$ and SMSe - regression estimate -0.32 (95\% Cl: -1.09 to 0.43 ), $p=0.360$, as opposed to volunteers where a 
positive association was evident - regression estimate 1.23 (95\% $\mathrm{Cl}$ : -2.20 to 4.67), $p=0.43$. Similar pattern was observed for the associations between WR and SMSd: patients with AF - regression estimate $-0.26(95 \% \mathrm{Cl}$ : -0.88 to 0.34$), \mathrm{p}=0.35$, and volunteers regression estimate 1.95 ( $95 \% \mathrm{Cl}:-2.66$ to 6.57 ), $\mathrm{p}=0.35$.

\section{Discussion}

Many studies have demonstrated the impact of the ANS imbalance in the initiation and maintenance of $\operatorname{AF}[2,11]$. The experimental data has shown the effect of disturbance of sympathetic innervation of the heart on the vulnerability of the left atrium for arrhythmogenic triggers arising from pulmonary veins [2].

Our study revealed a number of features of the scintigraphic patterns of total uptake and the regional distribution of neuronal tracer ${ }^{123}$ I-mIBG in the myocardium of the LV in healthy individuals and patients with paroxysmal AF.

Analysis of the regional uptake of ${ }^{123}$ I-mIBG in LV myocardium in healthy individuals and patients with $\mathrm{AF}$ demonstrates heterogeneity of the indicator distribution with the presence of sites of reduced uptake in the inferior wall and the apical region of the left ventricle. Similar data were obtained in a study by Gill et al. in which a lower degree of ${ }^{123}$ I-mIBG uptake in the area of the inferior wall and septum was noted in healthy volunteers compared to anteroposterior LV divisions [22]. The defects of ${ }^{123} \mathrm{I}$ mIBG uptake in the myocardium of the left ventricle were more common in patients with AF than in healthy volunteers. In this case, patients with AF had a more significant decrease in regional ${ }^{123}$ I-mIBG uptake in the delayed phase (SMSd). One of the possible explanations for this fact can be an increase in the tone of the sympathetic division of the ANS in response to a violation of the systolic function of the heart in the absence of a phase of atrial systole and the development of LV myocardial dysfunction. This hypothesis is confirmed by the results of a number of studies in which it has been established that even short-term myocardial ischemia can lead to so-called sympathetic cardiac remodelling $[23,24]$. Another explanation for the changes revealed is the development of dyssynchrony of myocardial contraction of the left ventricle in AF patients. In this case, as known, the inferior wall and septum are more sensitive than other segments of the LV myocardium, to a violation of the contraction sequence [25]. Therefore, in patients with a high frequency of AF paroxysms or with a longer duration of arrhythmia, it is possible to identify more significant regional pathological changes in sympathetic innervation of the LV myocardium.

In our study, between patients with AF and healthy volunteers, there was no significant difference in parameters such as the heart/mediastinum $(H / M)$ ratio and the washout rate (WR) of the tracer. In our opinion, this may be due to the small impact of the inferior wall and LV apex to the scintigraphic planar image of the heart, which reduces the difference in the severity of defects in radiopharmaceutical uptake in these areas of the myocardium. In addition, the results obtained could be affected by the form and duration of the AF. In the study of Grishaeva et al. [12] a disturbance of sympathetic activity of the myocardium, proportional to the duration of arrhythmia, was established in patients with a persistent AF.
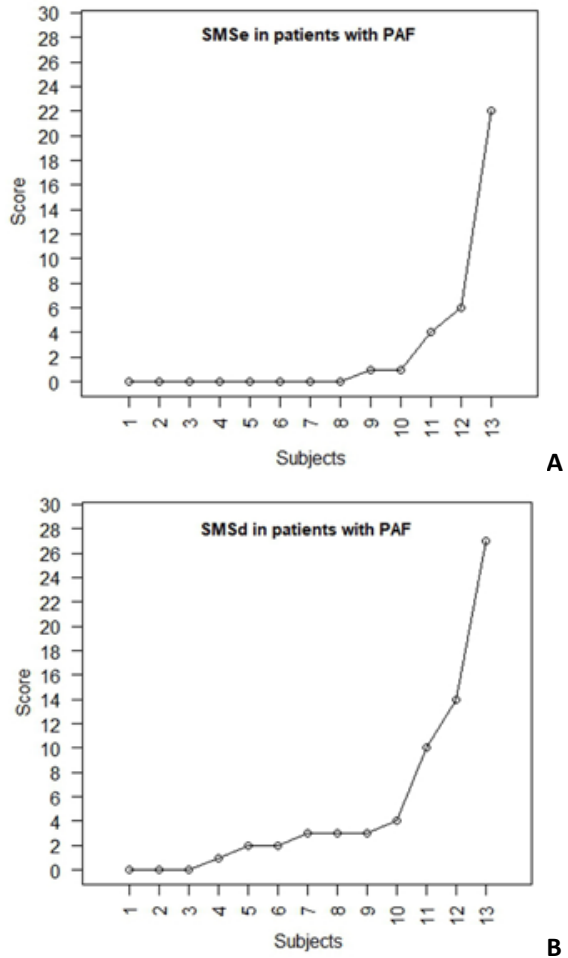

Figure 2. Early (A) and delayed (B) ${ }^{123} \mathrm{I}-\mathrm{mIBG}$ uptake defect score in patients with AF. SMSe, ${ }^{123}$ I-mIBG uptake defect score in early scans; SMSd, ${ }^{123}$ I-mIBG uptake defect score in delayed scans; PAF, patients with paroxysmal atrial fibrillation.
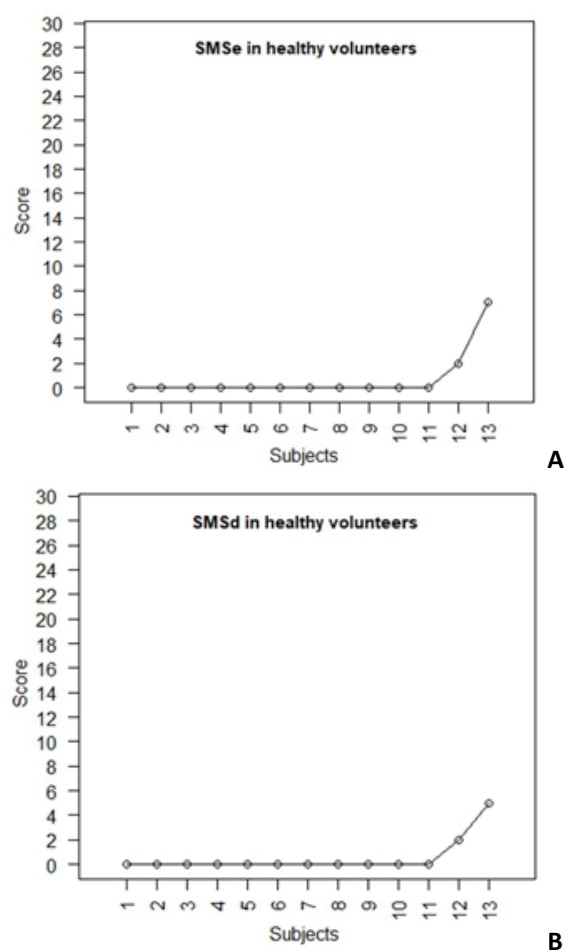

Figure 3. Early (A) and delayed (B) ${ }^{123} \mathrm{I}-\mathrm{mIBG}$ uptake defect score in healthy volunteers. SMSe, ${ }^{123}$ I-mIBG uptake defect score in early scans; SMSd, ${ }^{123}$ I-mIBG uptake defect score in delayed scans. 


\section{Conclusion}

Patients with paroxysmal AF have more significant regional changes in the sympathetic activity of the LV myocardium. Regional changes in sympathetic innervation may be early signs of an ANS imbalance in patients with AF and can probably be used to predict the course of the disease. Application of SPECT gamma camera with CZT-detectors allows obtaining qualitative tomographic slices of the myocardium and evaluating regional changes in sympathetic innervation of the heart.

\section{Limitations}

The limitations of the study stem from the relatively small sample size that may have resulted in imprecise estimates and lack of statistical power to detect differences within and between the groups.

Not withstanding the above limitations, it seems promising to analyze the regional distribution of ${ }^{123}$ I-mIBG in the myocardium of the left ventricle and near-cardiac structures for a non-invasive evaluation of the functioning of the ANS, predicting the course and choosing the optimal tactics for managing patients with various forms of $\mathrm{AF}$.

\section{Ethical approval}

All procedures performed in studies involving human participants were in accordance with the ethical standards of the institutional and/or national research committee and with the 1964 Helsinki declaration and its later amendments or comparable ethical standards.

\section{Funding}

This work was supported by a grant from the Russian Science Foundation No. 17-75-20118.

\section{Conflict if interest}

The authors declare that they have no other conflict of interest.

\section{References}

1. Benjamin EJ, Levy D, Vaziri SM, D'Agostino RB, Belanger AJ, Wolf PA Independent risk factors for atrial fibrillation in a population-based cohort. The Framingham Heart Study. JAMA 1994; 271(11): 840-844. https://dx.doi.org/10.1001/jama.1994.03510350050036.

2. Calkins H, Hindricks G, Cappato R, Kim YH, Saad EB, Aguinaga L, et al. 2017 HRS/EHRA/ECAS/APHRS/SOLAECE expert consensus statement on catheter and surgical ablation of atrial fibrillation. Heart Rhythm 2017; 14(10): 275 444. https://dx.doi.org/10.1016/j.hrthm.2017.05.012.

3. Scherlag B, Nakagawa H, Jackman W, Yamanashi WS, Patterson E, Po S. Electrical stimulation to identify neural elements on the heart: their role in atrial fibrillation. J Cardiovasc Electrophysiol 2005; 13(Suppl): 37-42. https://dx.doi.org/10.1007/s10840-005-2492-2.

4. Hou Y, Scherlag BJ, Lin J, Zhang Y, Lu Z, Truong K, et al. Ganglionated plexi modulate extrinsic cardiac autonomic nerve input: effects on sinus rate, atrioventricular conduction, refractoriness, and inducibility of atrial fibrillation. J Am Coll Cardiol 2007; 50(1): 61-68. https://dx.doi.org/10.1016/j.jacc.2007.02.066.

5. Schauerte P, Scherlag BJ, Pitha J, Scherlag MA, Reynolds D, Lazzara R, et al. Catheter ablation of cardiac autonomic nerves for prevention of vagal atrial fibrillation. Circulation 2000; 102(22): 2774-2780. https://dx.doi.org/10.1161/01.CIR.102.22.2774.

6. Amar D, Zhang $\mathrm{H}$, Miodownik S, Kadish A. Competing autonomic mechanisms precedes the onset of postoperative atrial fibrillation. $J$

\section{Am Coll Cardiol 2003; 42(7):}

1262-1268.

\section{https://dx.doi.org/10.1016/S0735-1097(03)00955-0.}

7. Ogawa M, Zhou S, Tan AY, Song J, Gholmieh G, Fishbein MC, et al. Left stellate ganglion and vagal nerve activity and cardiac arrhythmias in ambulatory dogs with pacing-induced congestive heart failure. J Am Coll Cardiol 2007; 50(4): 335-343. https://dx.doi.org/10.1016/i.jacc.2007.03.045.

8. Pokushalov E, Romanov A, Shugayev P, Artyomenko S, Shirokova N, Turov $A$, et al. Selective ganglionated plexi ablation for paroxysmal atrial fibrillation. Heart Rhythm 2009; 6(9): 1257-64. https://dx.doi.org/10.1016/j.hrthm.2009.05.018.

9. Katritsis DG, Pokushalov E, Romanov A, Giazitzoglou E, Siontis GC, Po SS, et al. Autonomic denervation added to pulmonary vein isolation for paroxysmal atrial fibrillation: a randomized clinical trial. J Am Coll Cardiol 2013; 62(24): 2318-25. https://dx.doi.org/10.1016/j.jacc.2013.06.053.

10. Erlandsson K, Kacperski K, van Gramberg D, Hutton BF. Performance evaluation of D-SPECT: A novel SPECT system for nuclear cardiology. Physics in Medicine and Biology 2009; 54(9): 2635-2649. https://dx.doi.org/10.1088/0031-9155/54/9/003.

11. Hou Y, Zhou Q, Po SS. Neuromodulation for cardiac arrhythmia. Heart Rhythm 2016; 13(2): 584-92. https://dx.doi.org/10.1016/j.hrthm.2015.10.001.

12. Wieland DM, Brown LE, Rogers WL, Worthington KC, Wu JL, Clinthorne $\mathrm{NH}$ et al. Myocardial imaging with a radioiodinated norepinephrine storage analog. J Nucl Med 1981; 22(1): 22-31. https://www.ncbi.nlm.nih.gov/pubmed/7452352.

13. Chen J, Wasmund SL, Hamdan MH. Back to the future: the role of the autonomic nervous system in atrial fibrillation. Pacing Clin Electrophysiol 2006; 29(4): 413-421. https://dx.doi.org/10.1111/i.1540-8159.2006.00362.x.

14. Somsen GA, Verberne HJ, Fleury E, Righetti A. Normal values and within-subject variability of cardiac I-123 MIBG scintigraphy in healthy individuals: implications for clinical studies. J NuclCardiol 2004; 11(2): 126-33. https://dx.doi.org/10.1016/i.nuclcard.2003.10.010.

15. Gimelli A, Liga R, Giorgetti A, Genovesi D, Marzullo P. Assessment of myocardial adrenergic innervation with a solid-state dedicated cardiac cadmium-zinc-telluride camera: first clinical experience. Eur Heart $J$ Cardiovasc Imaging 2014; 15(5): 575-585. https://dx.doi.org/10.1093/ehjci/jet258.

16. Gimelli A, Liga R, Genovesi D, Giorgetti A, Kusch A, Marzullo P. Association between left ventricular regional sympathetic denervation and mechanical dyssynchrony in phase analysis: a cardiac CZT study. Eur J Nucl Med Mol Imaging 2014; 41(5): 946-955. https://dx.doi.org/10.1093/ehjci/jet258.

17. Romanov A, Minin S, Breault C, Pokushalov E. Visualization and ablation of the autonomic nervous system corresponding to ganglionated plexi guided by D-SPECT 1231 -mIBG imaging in patient with paroxysmal atrial fibrillation. Clin Res Cardiol 2017; 106(1): 76-78. https://dx.doi.org/10.1007/s00392-016-1045-2.

18. Bellevre D, Manrique A, Legallois D, Bross S, Baavour R, Roth N, et al. First determination of the heart-to-mediastinum ratio using cardiac dual isotope (123/-MIBG/ ${ }^{99} \mathrm{mTC}$-tetrofosmin) CZT imaging in patients with heart failure: the ADRECARD study. Eur J Nucl Med Mol Imaging 2015; 42(12): 1912-1919. https://dx.doi.org/10.1007/s00259-0153141-3.

19. Cerqueira MD, Weissman NJ, Dilsizian V, Jacobs AK, Kaul S, Laskey WK, et al. Standardized myocardial segmentation and nomenclature for tomographic imaging of the heart: a statement for healthcare professionals from the Cardiac Imaging Committee of the Council on Clinical Cardiology of the American Heart Association. Circulation 2002; 105(4): 539-542. https://dx.doi.org/10.1161/hc0402.102975.

20. Gill JS, Hunter GJ, Gane G, Camm AJ. Heterogeneity of the human myocardial sympathetic innervation: in vivo demonstration by iodine 123-labeled meta-iodobenzylguanidine scintigraphy. Am Heart J 1993; 126(2): 390-398. https://dx.doi.org/10.1016/0002-8703(93)91056-K. 
21. Lishmanov YuB, Saushkina YuV, Minin SM, Efimova IYu, Kisteneva IV, Popov SV. Scintigraphic estimation of the sympathetic innervation of the heart and myocardial perfusion in patients with atrial fibrillation. Russ J Cardiol 2014; 12(6): 13-18. https://dx.doi.org/10.15829/15604071-2014-12-13-18.

22. Grishaev SL, Tkachenko KN, Svistov AS, Nikiforov VS, SukhovVYu. Potentialities of single photon emission computed tomography in assessment of sympathetic myocardial activity in patients with chronic atrial fibrillation. Journal of Arrhythmology 2011; (63): 12-15. Russian. https://elibrary.ru/item.asp?id=21003600.

23. Simantirakis EN, Prassopoulos VK, Chrysostomakis SI, Kochiadakis GE, Koukouraki SI, Lekakis JP, et al. Effects of asynchronous ventricular activation on myocardial adrenergic innervation in patientswith permanent dual-chamber pacemakers; an I(123)metaiodobenzylguanidine cardiac scintigraphic study. Eur Heart J 2001; 22(4): 323-332. https://dx.doi.org/10.1053/euhj.2000.2482.

24. Losik DV, Romanov AB, Shabanov VV, Bairamova SA, Yakubov AA, Stenin IG. Progression of atrial fibrillation after a failed initial ablation procedure in patients with paroxysmal atrial fibrillation: a randomized comparison of antiarrhythmic drug therapy vs re-ablation. Patologiya Krovoobrashcheniya I Kardiokhirurgiya (Circulation Pathology and Cardiac Surgery) 2015; 19(2): 84-92. Russian. https://dx.doi.org/10.21688/1681-3472-2015-2-84-92.

\section{Authors:}

Stanislav M. Minin - MD, PhD, Head of the Radioisotope Diagnosis Department, Meshalkin National Medical Research Center, Novosibirsk, Russia. http://orcid.org/0000-0001-6626-6408.

Nikita A. Nikitin - MD, Radiologist, Radiology Department, Meshalkin National Medical Research Center, Novosibirsk, Russia. http://orcid.org/0000-0001-5643-9109.

Vitaly V. Shabanov - Meshalkin National Medical Research Center, Novosibirsk, Russia. http://orcid.org/0000-0001-9066-3227.

Denis V. Losik - Meshalkin National Medical Research Center, Novosibirsk, Russia. http://orcid.org/0000-0003-4886-9648.

Igor L. Mikheenko - Meshalkin National Medical Research Center, Novosibirsk, Russia. http://orcid.org/0000-0002-3552-7158.

Dmitry N. Ponomarev - Meshalkin National Medical Research Center, Novosibirsk, Russia. http://orcid.org/0000-0003-3639-7444.

Evgeny A. Pokushalov - Meshalkin National Medical Research Center, Novosibirsk, Russia. http://orcid.org/0000-0002-2560-5167.

Alexander B. Romanov - Meshalkin National Medical Research Center, Novosibirsk, Russia. http://orcid.org/0000-0002-6958-6690. 